Annuaire suisse de politique de développement

Partenariats public-privé et coopération internationale

\title{
Le concept de " partenariat public-privé » est-il bien posé ? La coresponsabilité de l'économie privée en politique de développement
}

Peter Ulrich et Florian Wettstein

\section{OpenEdition}

Journals

Édition électronique

URL : http://journals.openedition.org/aspd/339

DOI : 10.4000/aspd.339

ISSN : 1663-9669

Éditeur

Institut de hautes études internationales et du développement

Édition imprimée

Date de publication : 1 octobre 2005

Pagination : 43-55

ISSN : $1660-5934$

\section{Référence électronique}

Peter Ulrich et Florian Wettstein, « Le concept de « partenariat public-privé » est-il bien posé ? La coresponsabilité de l'économie privée en politique de développement ", Annuaire suisse de politique de développement [En ligne], 24-2 | 2005, mis en ligne le 18 février 2010, consulté le 08 septembre 2020. URL : http://journals.openedition.org/aspd/339; DOI : https://doi.org/10.4000/aspd.339 


\title{
Le concept de "partenariat public-privé " est-il bien posé ? La coresponsabilité de l'économie privée en politique de développement
}

\author{
Peter Ulrich* et Florian Wettstein**
}

orsqu' on aborde un nouveau sujet, les concepts à la mode peuvent se révéler utiles ou dangereux. Utiles par leur pouvoir de capter l'attention d'un large public encore peu au fait d'une thématique particulière. Dangereux souvent pour cause de polysémie, en particulier avec les anglicismes, et pour cause de propagation incontrôlée alors même que leur contenu reste flou. A cet égard, le «partenariat public-privé» ne constitue pas une exception. On l'applique à une multitude d'interactions entre acteurs publics et acteurs privés, trop souvent sans s'être penché sur les hypothèses et implications normatives de la collaboration postulée. Hypothèses et implications qu'il s'agit pourtant d'étudier si l'on entend concrétiser le modèle (toujours vague) et lui ouvrir l'ensemble du domaine d'application auquel il prétend. C'est dans cet esprit que nous souhaitons examiner ici la contribution que le «partenariat» entre les autorités et l'économie privée peut apporter à la politique de développement.

\section{En préalable à l'étude du concept: de l'opposition public-privé...}

Un «partenariat», voilà qui est toujours une bonne chose, non? Les partenariats public-privé bénéficient donc, du moins aux yeux de leurs avocats, d'un préjugé positif: ils font sens, ils sont légitimes. Sans même en avoir débattu, on en réduit l'horizon normatif à une offre plus efficace en services publics, plus efficace que ce que le secteur public pourrait fournir de son seul chef. Ce faisant, on oublie que les problèmes politico-économiques complexes ne peuvent être résolus que sur la base d'idées directrices normatives d'une bonne pratique, explicitement justifiées ou simplement tacitement admises. Le discours sur la création de partenariats public-privé dans le domaine de la politique de développement recèle son lot de «présupposés normatifs» tacites sur ce qu'est le bon développement socio-économique. Eclairer ces présupposés et en vérifier tant le sens que la légitimité est une des tâches de l'éthique économique'.

* Docteur en sciences politiques, professeur titulaire en éthique économique à l'Université de SaintGall, où il dirige l'Institut d'éthique économique.

** Licencié en sciences économiques de l'Université de Saint-Gall, où il est collaborateur scientifique et doctorant à l'Institut d'éthique économique; international research fellow pour le «Program on Human Rights and Justice» du Massachusetts Institute of Technology (MIT).

1 L'éthique économique telle que nous l'entendons ici est décrite par exemple dans P. Ulrich, Integra-

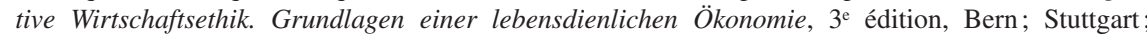
Wien, Haupt, 2001, pp. 95 et suiv. 
En matière de partenariat public-privé, on trouve de tels présupposés implicites normatifs dès l'introduction de la dichotomie public-privé. Il est significatif que ses défenseurs comme ses critiques posent comme point de départ de leur réflexion la séparation des domaines public et privé. En saluant le «partenariat», on entérine l'existence de deux acteurs bien distincts mais de même niveau, aux orientations opérationnelles différentes, pouvant se rencontrer utilement sur un objectif commun (lequel ?). Ce faisant, on présuppose visiblement l'existence de systèmes de motivations qu'il s'agit, pour aboutir, de ramener à un dénominateur commun. Les critiques eux aussi se réfèrent à cette dichotomie, mais en se battant au contraire pour que soit respecté le strict respect de la frontière entre ces deux sphères ${ }^{2}$.

On le voit, d'emblée se pose la question de ce qu'on entend par partenariat public-privé. Cette forme de «création de valeur» est-elle donc si extraordinaire qu'il lui faille une dénomination particulière? Selon l'argumentation de ses avocats comme de ses critiques, le secteur public serait donc l'adversaire du secteur privé et vice-versa. Cet accord ne doit rien au hasard étant donné que d'un côté, tout ce qui évoque de près ou de loin le secteur privé est quasi automatiquement diabolisé, et que de l'autre, en particulier dans les milieux bourgeois de droite, on manifeste un ressentiment croissant contre tout ce qui ressortit à l'Etat, un Etat qu'on cherche alors à maintenir le plus possible à l'écart. Les campagnes du type «plus de liberté, moins d'Etat» illustrent bien cette lecture idéologisée des secteurs public et privé. Selon ce schéma, l'idée de secteur public est automatiquement associée à celle de contrainte. L'un dans l'autre, l'Etat serait l'adversaire du secteur privé, lequel incarnerait la liberté personnelle illimitée. L'instance garante de cette liberté illimitée serait - le hasard n'y est pour rien - le marché «libre», libre de toutes interférences étatiques et des distorsions qui en découlent. Donc un marché idéalisé. L'image même de la liberté individuelle et, partant, d'un ordre social libéral porté au pinacle. Lequel ordre trouve son aboutissement dans une société tout entière de marché où la liberté privée aurait congédié la contrainte publique et où la «main invisible» d'Adam Smith se chargerait de tout.

\section{. au partenariat public-privé}

Il se trouve que la liberté peut être comprise autrement que comme la simple absence de contrainte étatique. La liberté bien comprise est toujours liée à la liberté publique parce que son opposé - l'arbitraire - se définit par le bon plaisir privé. C'est pourquoi, dans la foulée de sa critique du pouvoir arbitraire, Emmanuel Kant reconnaît la «liberté illimitée d'user de sa propre raison », c'est-à-dire de se former ses propres motifs d'action et de les justifier en «usant publiquement de sa raison $»^{3}$. Autrement dit, la liberté légitime d'agir selon ses propres motifs implique que ces motifs soient publiquement défendables. La liberté ne doit donc pas être comprise comme liberté individuelle, mais comme liberté générale, c'est-à-dire la plus grande liberté de tous. Dans cette perspective, elle

2 Voir par exemple J. Richter, «We the Peoples» or «We the Corporations»? Critical Reflections on UN-business «Partnerships», Geneva, International Baby Food Action Network (IBFAN); Geneva Infant Feeding Association (GIFA), 2003, p. 9.

3 E. Kant, «Beantwortung der Frage: Was ist Aufklärung?», in W. Wieschedel (dir.), Immanuel Kant Werkausgabe, vol. 11, 6e édition, Frankfurt a. M., Suhrkamp, 1982, p. 57. 
apparaît comme essentiellement de nature politico-éthique ${ }^{4}$. Le politique ne limite pas simplement la liberté, il la rend au contraire possible et l'élargit. Il n'y a rien de plus public que la définition et la délimitation de ce qui, dans une société, est chose privées.

Mais si le domaine privé ne peut se constituer que par légitimation publique, il n'est rationnellement plus possible de raisonner comme si les deux sphères publique et privée étaient entièrement distinctes. La raison purement privée et non validée par l'usage public ne saurait ainsi être invoquée comme principe d'action. Ce n'est donc pas par leur engagement dans des partenariats publicprivé que les entreprises accèdent au statut d'acteurs publics, elles le sont par définition. Et les grandes entreprises doivent toujours être comprises comme des institutions quasi publiques ${ }^{6}$.

C'est pourquoi nous défendons dans cette contribution une interprétation du partenariat public-privé qui ne s'arrête pas à la séparation dogmatique entre les domaines public et privé. Nous en proposons une vision alternative et clarifiée qui ne rejette pas globalement toute collaboration entre le public et le privé, mais y voit au contraire un instrument très prometteur de la politique de développement. Ce point de vue, qu'il s'agit d'approfondir, ne confond pas partenariats public-privé et privatisation forfaitaire du secteur public, pas plus qu'il ne réduit ces partenariats à une simple éthique du don, comme cela a souvent été le cas. La critique de ces deux visions trop courtes des choses nous servira de base au développement d'une alternative permettant de les intégrer.

\section{L'interprétation économiste: les partenariats public-privé comme la privatisation graduelle du secteur public}

Il est rare d'entendre expliquer que la collaboration entre secteurs public et privé est souhaitable afin de permettre aux entreprises de s'imprégner de l'esprit fonctionnaire. On évoque en général l'inverse: le secteur public aurait beaucoup à apprendre du monde de l'entreprise, de sa force d'innovation et de son efficacité. Et, pour le secteur privé, il s'agit à cette occasion de reconquérir une part du terrain qui lui a été retiré de manière injustifiée par les pouvoirs publics. Cette «restitution» de tâches publiques au secteur privé n'est pas présentée comme une stratégie de politique sociale et économique, mais bien comme une cure d'assainissement de l'appareil d'Etat, prescrite pour des raisons quasi métaphysiques, en vue de renforcer l'économie nationale pour affronter la concurrence internationale: «Nous devons par conséquent trouver de nouveaux moyens de faire profiter le secteur public des gains de productivité enregistrés ces dernières années dans le secteur privé et - en même temps - faire participer le secteur privé de manière appropriée à l'exécution des tâches réservées jus-

4 Voir l'étude détaillée de R. Dworkin, Bürgerrechte ernst genommen, Frankfurt a. M., Suhrkamp, 1984, pp. 429 et suiv.

5 Au sujet de cette interprétation du libéralisme, voir P. Ulrich, Integrative Wirtschaftsethik, $3^{\mathrm{e}}$ édition, Bern; Stuttgart; Wien, Haupt, 2001, pp. 324 et suiv., particulièrement p. 315. P. Ulrich, Der entzauberte Markt. Eine wirtschaftsethische Orientierung, Freiburg i. B., Herder, 2002, pp. 72 et suiv. T. Maak, Die Wirtschaft der Bürgergesellschaft, Bern; Stuttgart; Wien, Haupt, 1999, pp. 206 et suiv.

6 Voir P. Ulrich, Die Grossunternehmung als quasi-öffentliche Institution. Eine politische Theorie der Unternehmung, Stuttgart, C.E. Poeschl, 1977. 
qu'alors à l'Etat. $\gg^{7} \mathrm{La}$ course globale à la productivité apparaît donc comme une force salutaire qui impose aux gouvernements nationaux de restituer les espaces d'action privés indûment occupés. C'est ainsi que s'impose cette «politique posée comme une obligation $»^{8}$, même «dans la douleur $»^{9}$, car, en dernière analyse, c'est en assurant la compétitivité qu'on assure la croissance économique. Laquelle est considérée à son tour comme le garant, voire le synonyme de l'élévation durable du niveau de vie général et donc comme l'essence normative de toute politique de développement bien comprise.

Dans le domaine de la politique de développement, la fonction des partenariats public-privé est également interprétée dans ce cadre purement économiste ${ }^{10}$. Il s'agirait en premier lieu de faire progresser la privatisation du secteur public et de stimuler ainsi la croissance. Ce qui expliquerait l'importance accrue que, depuis un certain temps, les organisations de développement, en particulier la Banque mondiale, accordent dans leurs programmes aux partenariats publicprivé. Dans les secteurs traditionnellement gérés par les collectivités publiques comme, par exemple, l'approvisionnement en eau, cet alignement progressif sur la logique de la privatisation et de la concurrence permet de renoncer ${ }^{11}$ aux thérapies de choc discréditées des architectes du consensus de Washington.

On attend des partenariats public-privé qu'ils assument les tâches publiques avec plus d'efficacité et d'inventivité. Le facteur qui en serait le garant est le profit qu'en escomptent les entreprises participantes ${ }^{12}$. Le motif du «profit» devient donc le facteur décisif de la réussite des partenariats. Si l'on considère que les domaines public et privé, avec leurs objectifs et leurs motivations respectifs, sont fondamentalement distincts, le partenariat n'aboutira donc que si l'entreprise privée peut compter sur une augmentation de ses gains grâce à la prise en charge de la tâche publique, ce qui réduit quasiment le secteur public au statut de nouveau segment de marché. Mais si l'on ne recourt aux partenariats public-privé que dans des situations win-win (c'est-à-dire dans lesquelles il n'y a que des gagnants et pas de perdants), il en découle que l'économie privée ne peut être invitée comme partenaire que là où le motif du profit peut contribuer à satisfaire des objectifs publics. Ce qui soulève deux objections décisives du point de vue de l'éthique économique.

Lorsque, pour accroître la présence du secteur privé dans les affaires publiques, on explique que la complexité des problèmes (globaux) actuels dépasse les dif-

7 T. Mirow, «Public-Private Partnership - eine notwendige Strategie zur Entlastung des Staates», in D. Budäus et P. Eichhorn (dir.), Public-Private Partnership. Neue Formen öffentlicher Aufgabenerfüllung, Baden-Baden, Nomos Verlagsgesellschaft, 1997, p. 15.

8 Voir U. Thielemann, Freiheit unter den Bedingungen des Marktes. Oder doch gegenüber der Marktlogik? Vom verfehlten Umgang mit Sachzwängen, Berichte des Instituts für Wirtschaftsethik, $\mathrm{n}^{\circ} 101$, St. Gallen, Institut für Wirtschaftsethik, 2004, pp. 3 et suiv.

9 T. Mirow, op. cit., p. 15.

10 Sur l'économisme en politique de développement: P. Ulrich, «Was ist "gute" sozio-ökonomische Entwicklung? Eine wirtschaftsethische Perspektive», Zeitschrift für Wirtschafts- und Unternehmensethik, vol. $5, \mathrm{n}^{\circ} 1,2004$, pp. 8 et suiv.

11 Voir à ce sujet F. Wettstein, Die «unsichtbare Hand» des Wassermarktes. Zur Privatisierungs- und Wettbewerbslogik im Wasserversorgungssektor, Berichte des Instituts für Wirtschaftsethik, $\mathrm{n}^{\circ}$ 103, St. Gallen, Institut für Wirtschaftsethik, 2004.

12 E.S. Savas, Privatization and Public-Private Partnerships, New York; London, Chatham House Publishers, 2000, pp. 240 et suiv. 
férents acteurs publics ${ }^{13}$, le critère win-win ne peut être invoqué ni comme condition nécessaire ni comme condition suffisante. En effet, si, dans l'exécution du mandat public, l'entreprise privée se rend progressivement de plus en plus indispensable, la responsabilité qui lui échoit l'éloigne de son rôle historique et sociétal original en lui retirant sa motivation première qui est le profit économique. La condition win-win est de toute manière insuffisante car l'ensemble des problèmes à la résolution desquels les acteurs privés pourraient potentiellement contribuer ne présente de loin pas un potentiel de gains attrayant. Vouloir l'ignorer serait s'accrocher au credo métaphysique d'un marché qui résoudrait automatiquement tous les problèmes humains. Là où les problèmes sont les plus tenaces et les plus graves, les perspectives de profit manquent, si bien que là où l'engagement serait le plus nécessaire, on ne pourrait par définition pas créer de partenariats, ou alors seulement des partenariats condamnés à l'inefficacité. Les partenariats motivés uniquement par la logique win-win et donc, pour les entreprises, par un calcul économique se révèlent ainsi d'emblée insuffisants pour résoudre les problèmes publics.

Vouloir résoudre les goulets d'étranglement du secteur public en faisant jouer les intérêts particuliers privés, c'est bien souvent introduire le loup dans la bergerie. En effet, de nombreux problèmes sociétaux proviennent justement du manque d'intégration sociale du système économique, de sa course sans frein au profit. Poser qu'on obtiendra une croissance économique à long terme en acceptant des sacrifices à court terme est un paralogisme car les sacrifices à accepter sont inhérents à la logique économique. Ce dont on a besoin pour réussir les partenariats entre secteur public et secteur privé, ce n'est pas la simple privatisation du secteur public. Celle-ci assujettirait l'exécution des tâches publiques à une logique marchande partisane et éliminerait simultanément les mécanismes compensatoires par lesquels les pouvoirs publics cherchent à assurer une certaine équité; elle ne mesurerait l'engagement des entreprises privées dans le secteur public que sur la base de leurs performances financières et en les déchargeant de toute responsabilité sociale. Ce serait progressivement dissoudre le lien essentiel entre équité et affaires publiques. Interprétés correctement, les partenariats public-privé doivent au contraire être vus comme d'excellentes constructions permettant le transfert partiel de tâches publiques et de la (co)responsabilité y attenant vers le secteur privé. Le point crucial d'un partenariat ainsi compris est justement le renversement des priorités entre le profit économique privé et la légitimité publique. Les partenariats public-privé offrent de la sorte à l'entreprise une possibilité de concrétiser sous forme intégrée la responsabilité publique qui lui incombe de toute manière en tant qu'acteur économique privé et donc en tant qu'institution «quasi publique».

13 Comme par exemple la directrice générale de l'Organisation mondiale de la santé (OMS), Gro Harlem Brundtland, au sujet de l'intégration d'entreprises privées dans les stratégies sanitaires de l'OMS. Voir G.H. Brundtland, Address by Dr. Gro Harlem Brundtland, Director-General, to the Fifty-Fifth World Health Assembly, Geneva, World Health Organization, 13 May 2002, <www.who.int/genomics/ speeches/en> (dernière consultation 16 janvier 2005). 


\section{L'interprétation «éthique du don » : la philanthropie entrepreneuriale} comme passerelle entre les sphères publique et privée

On pense souvent pouvoir mesurer la responsabilité sociale des entreprises à la largesse de leurs dons. Cette conception erronée se répercute jusque dans la discussion sur le partenariat public-privé. Et il est vrai que, à y regarder de près, de nombreux partenariats public-privé se révèlent n'être pas grand-chose de plus qu'une aide au financement d'institutions publiques par des entreprises privées.

Dans ces cas, on constate que la délégation cohérente des questions de politique de développement aux mécanismes du marché n'est que partiellement ou pas du tout réalisée. Le développement, tel qu'on le souhaite, échappe en bonne partie aux mécanismes du marché, lesquels doivent dans ce domaine être complétés ou redressés par des mesures philanthropiques. Ces mesures correctives restent purement complémentaires, ne portant que sur les résultats de la coordination du marché et ne touchant pas aux mécanismes mêmes de la coordination. Les entreprises et leurs activités commerciales ne sont donc exposées à aucune exigence d'éthique de développement. Alors que, dans la variante économiste du partenariat public-privé, les aspects éthiques du développement sont entièrement traduits en catégories économiques, on construit ici une sorte de «conception des deux mondes » selon laquelle - du moins le présume-t-on - s'affrontent une logique économique neutre en matière de développement et l'éthique caritative qui s'en trouve détachée ${ }^{14}$. Ce qui correspond malheureusement à une vision dépassée de l'aide au développement, qui lutte contre les symptômes sans réfléchir à leurs causes politico-économiques. On ignore ainsi le fait que la logique économique du profit individuel ne fait pas toujours partie de la solution et qu'elle fait même parfois partie du problème.

Et puis, dans la vision du partenariat public-privé réduit à l'éthique du don, le fait qu'une entreprise puisse se montrer d'autant plus généreuse que ses profits ont été considérables transforme la maximisation du profit en condition préalable tacite d'accession au rôle de partenaire généreux. Dans cette variante, la distinction entre tâches publiques et privées conduit visiblement au partage de la problématique des entreprises: la coresponsabilité publique et politique des entreprises s'arrête au juste emploi (d'une partie) de leurs profits, alors que la manière dont ces profits sont acquis reste ignorée ${ }^{15}$. Une interprétation du partenariat public-privé qui réglerait ainsi la question du sens et de la finalité du travail des entreprises dans les pays en développement est, du point de vue de l'éthique du développement, impensable.

Ce qui reste visiblement inexprimé dans cette version du partenariat publicprivé, c'est le lien objectif entre les stratégies de maximisation des profits de l'économie privée et la prise en charge de responsabilités dans le domaine de la politique de développement. Une pareille collaboration entre l'économie privée et le secteur public reposerait sur ce qui va de soi pour les entreprises, c'està-dire que la prise en charge de responsabilités sociales et publiques est un élément intégral de leur légitime effort de maximisation des bénéfices et non pas

\footnotetext{
14 Pour creuser la «conception des deux mondes », voir P. Ulrich, Integrative Wirtschaftsethik. Grundlagen einer lebensdienlichen Ökonomie, op. cit., pp. 102 et suiv.

15 Voir à ce sujet ibid., pp. 421 et suiv.
} 
seulement une «indulgence» leur permettant de soulager leur éventuelle mauvaise conscience par des dons éthiques ciblés.

Mais alors, comment faut-il concevoir les partenariats public-privé, comment les ancrer dans une perspective d'éthique de développement? Nous tentons ci-dessous de le faire sur la base du «bon» développement socio-économique et en précisant en quoi consiste dans ce domaine la coresponsabilité des entreprises.

\section{L'interprétation intégrante: les partenariats public-privé comme expression de la coresponsabilité de l'entreprise en politique de développement}

Du point de vue de l'éthique, il est un peu court de réagir aux nombreuses questions que soulève le développement socio-économique par une seule et même réponse: laisser jouer les mécanismes du marché. Des marchés fonctionnant librement sont un des moyens du bon développement, et non sa fin. Il faut leur donner un cadre normatif approprié et des structures incitatives. Ce qui signifie que la politique moderne de développement ne doit pas être paternaliste, mais au contraire émancipatrice. Il s'agit de donner à un maximum d'individus la possibilité de mener dans la dignité et le respect une vie «pleine d'aspirations», comme le formule le Prix Nobel d'économie Amartya Sen, principal économiste du développement actuel ${ }^{16}$. "L'empowerment plutôt que l'aumône», comme le formule la Direction du développement et de la coopération suisse (DDC). Il s'agit bien là de liberté, pas uniquement de la «liberté du marché» mais d'une réelle liberté civique partagée par tous les citoyens. Le programme $\mathrm{du}$ «libéralisme républicain ${ }^{17}{ }^{17}$ est de garantir cette liberté par l'Etat de droit et d'en faire une réalité politico-économique.

Par «développement», on entend le processus complet de transformation sociale vers une société citoyenne d'individus égaux et libres, conscients du rôle constitutif de la res publica et de l'importance de l'ordre public pour les libertés individuelles, et qui s'en sentent donc coresponsables de manière engagée. De ce point de vue, le bon développement est un projet complet de civilisation, avec ses aspects politico-culturels, juridiques et socio-économiques. Il s'agit là d'un projet universel, qui ne retient pas les distinctions habituelles entre pays développés et pays sous-développés, souvent unilatéralement fondées sur des indicateurs économiques. Selon ce point de vue, aucun pays du monde n'est exclu du développement. On voit bien aujourd'hui dans les pays occidentaux «hautement développés » quelle désintégration sociale une croissance unilatéralement économique peut entraîner. A beaucoup d'égards, ces pays doivent être considérés comme des pays en développement au sens propre du terme. Ce qui ne signifie pas que le développement soit complètement découplé de la croissance économique. Presque personne ne conteste que l'économie est à la fois l'un des éléments et l'un des instruments nécessaires aux sociétés citoyennes épanouies ${ }^{18}$. Le «bon» développement socio-économique ayant pour objectif

16 A. Sen, Un nouveau modèle économique. Développement, justice, liberté, Paris, Odile Jacob, 1999. Le titre de l'original est plus évocateur: Development as Freedom.

17 Sur le concept de libéralisme républicain et ce qui le distingue du simple libéralisme économique, voir P. Ulrich, Integrative Wirtschaftsethik. Grundlagen einer lebensdienlichen Ökonomie, op. cit., pp. 293 et suiv.

18 Voir P. Niggli, Nach der Globalisierung. Entwicklungspolitik im 21. Jahrhundert, Zürich, Rotpunktverlag, 2004, p 57. 
d'être socialement utile, on ne saurait en mesurer la contribution uniquement par des critères économiques (risque d'économisme de la politique de développement).

Au sein d'une telle économie, tout orientée vers le service de la vie, la coresponsabilité des entreprises en matière de politique de développement ne se manifeste ni dans la maximisation du profit ni dans les activités caritatives. Les entreprises devraient au contraire se sentir tout entières engagées dans la société, se voir comme des acteurs publics complets et endosser la coresponsabilité républicaine que cela implique. En tant que corporate citizens, global players et bons citoyens du monde, elles devraient reconnaître que leur «mission» est de contribuer au progrès de la civilisation de leurs pays hôtes, et cela non pas uniquement en réalisant leurs objectifs économiques privés dans le respect des règles du bien-être général (réduction économiste), ni en plus de leurs activités commerciales «normales» (réduction caritative), mais sur la base d'une politique éthique intégrée des affaires ${ }^{19}$.

Il en ressort que les organisations (pas si) «privées» sont publiquement coresponsables et en tant que telles elles interprètent les partenariats public-privé non comme de simples extensions commerciales ou caritatives, mais bien comme l'occasion d'exercer cette coresponsabilité. Le partenariat avec des instances publiques peut s'avérer fructueux dans la mesure où, justement, les deux partenaires sont engagés dans un processus d'apprentissage, et non pas seulement les institutions publiques qui, selon la conception dominante, auraient besoin de se frotter aux entreprises. Les acteurs publics, comme par exemple les responsables du développement, disposent d'un grand savoir-faire et d'une riche expérience. Ils savent ainsi, alors que l'économie l'a presque entièrement oublié, jouer leur rôle de manière cohérente au sein et au service de la société. Il est possible que les institutions publiques aient quelques leçons d'efficacité à prendre du côté de l'économie, mais celle-ci peut également découvrir auprès d'elles quelques nouvelles vues sur l'approche éthique des attentes sociales légitimes et la gestion des nombreux points de vue souvent conflictuels qui en sont la source. Les entreprises peuvent ainsi acquérir une meilleure compréhension des problèmes délicats de bonne organisation publique et de la «bonne» gestion d'entreprise au-delà des seules contraintes économiques. On a récemment montré que la société civile - donc les citoyens et les citoyennes euxmêmes - souhaitait de plus en plus explicitement que les entreprises jouent leur rôle et prennent leurs responsabilités de manière "civilisée », c'est-à-dire dans le sens du corporate citizenship, face aux problèmes de société, parmi lesquels la politique de développement ${ }^{20}$.

Une telle évolution relève-t-elle de l'idéalisme utopique? On renverra ceux qui le craignent aux exemples bien documentés qui montrent le contraire. Il existe déjà plusieurs partenariats public-privé visant réellement à l'intégration politique coresponsable des entreprises dans le policy-making et dans les processus publics de décision. Citons, par exemple dans le secteur pharmaceutique, les

19 Sur les critères et les éléments d'une telle politique intégrée des affaires, voir P. Ulrich, Integrative Wirtschaftsethik. Grundlagen einer lebensdienlichen Ökonomie, op. cit., pp. 427 et suiv.

20 Voir à ce sujet Y. Lunau et F. Wettstein, Die soziale Verantwortung der Wirtschaft. Was Bürger von Unternehmungen erwarten, Bern; Stuttgart; Wien, Haupt, 2004. 
collaborations avec des instances officielles telles que l'OMS, qui dépassent fréquemment le simple don de produits pour viser clairement l'organisation participative de dispositions-cadres en matière de santé publique. On peut naturellement spéculer sur les motivations de certains participants (hidden agendas) et mettre le doigt sur de nombreuses incohérences, mais ces exemples laissent bien présager de l'avenir. L'exemple le plus marquant visant aux changements de l'économie globale évoqués ci-dessus est sans doute le UN Global Compact. Etant donné que le Global Compact incarne cette nouvelle forme de collaboration public-privé à visée politique coresponsable sous une forme quasiment pure, on le perçoit rarement comme un exemple typique de partenariat publicprivé (pratiqué, qui plus est, de manière incohérente). C'est pourtant ce type de partenariats de haut vol $^{21}$ qui a le plus de chance d'être porté par des global players et d'aboutir à des pratiques commerciales raisonnables et socialement responsables. La condition est que la société civile accueille ce «pacte pour une nouvelle pensée» avec suffisamment de «sympathie critique», comme le réclame Thomas Fitschen, du Département des affaires extérieures à Berlin ${ }^{22}$. C'est ce que nous allons faire, en soumettant le Global Compact des Nations unies à une courte analyse empreinte de sympathie critique dans le cadre de l'horizon normatif esquissé ci-dessus.

\section{Exemple pratique de partenariat public-privé intégrateur: le Global Compact de l'ONU}

En 1999, le secrétaire général de l'ONU, Kofi Annan, lançait un «pacte mondial», le Global Compact, dont l'objectif est d'obtenir que les entreprises transnationales s'engagent sur une base volontaire à respecter neuf principes universels dans le domaine du travail, des droits de l'homme et de l'environnement. Ce catalogue a récemment été complété par un dixième principe visant la lutte contre la corruption.

Ce qui distingue ce type de partenariat entre l'ONU et les entreprises transnationales des partenariats public-privé habituels est l'accent mis sur l'élaboration de normes et de principes par le dialogue et l'apprentissage communs. Alors que les partenariats habituels à objectif sociétal ne présentent qu'un caractère temporaire, le Global Compact se pose comme une plate-forme durable d'apprentissage et d'élaboration de normes de bonne conduite des entreprises dans une perspective générale de progrès de la civilisation et de politique de développement. Soumise à ce grand principe régulateur, l'initiative vise la transformation à long terme de la culture globale de la concurrence. Il s'agit donc d'ériger un ordrecadre (sub)politique permettant aux entreprises soumises à une pression concurrentielle toujours croissante de travailler de manière socialement responsable.

La dichotomie entre public et privé est ici aussi adoucie. S'engager à respecter les dix principes du Global Compact, c'est bien remplacer la morale privée du

21 J. Richter, Public-Private Partnerships and International Health Policy Making: How Can Public Interests Be Safeguarded?, Helsinki, Ministry for Foreign Affairs of Finland, 2004, p. 74.

22 Au sujet de ces deux expressions, voir T. Fitschen, Der «Global Compact» als Zielvorgabe für verantwortungsvolles Unternehmertum. Idee mit Zukunft oder Irrweg für die Vereinten Nationen?, Diskussionspapier, n 04-8, Wittenberg, Wittenberg-Zentrum für Globale Ethik, 2004, pp. 7 et 10. 
marché par le devoir de rendre publiquement des comptes de ses activités, c'està-dire de soumettre la course au profit à des principes publics de légitimité. Cela passe par l'engagement commun des entreprises de respecter les règles d'une saine concurrence, qui tienne compte des droits imprescriptibles de la dignité humaine. On peut espérer de la sorte briser l'absurde spirale de la concurrence globale débridée dans laquelle les entreprises s'interdisent toute considération pour les facteurs «extraéconomiques» de nature humaine, sociale et écologique. C'est pour cela que plaident le spiritus rector de ce Pacte mondial, John Gerard Ruggie, ainsi que le directeur du Global Compact Office de New York, George Kell, qui y voient l'instrument permettant de "close the gap between the strictly economic sphere and the broader public agendas that exists at the global level today, which the corporate sector itself created $»^{23}$. C'est pourquoi la fixation de règles du jeu généralement contraignantes est un acte collectif d'intelligence entrepreneuriale, les corporate citizens réellement prêts à assumer leur responsabilité sociale ne souhaitant pas se trouver face à des concurrents qui s'obstinent à les ignorer. Obtenir que le plus de concurrents possible suivent des règles de comportement éthiquement défendables, c'est civiliser la concurrence. Le terme «civiliser» prend là toute sa valeur selon laquelle les acteurs économiques intègres ne dissocient par leur recherche du succès privé de leur civisme républicain mais y voient une quête commune. C'est bien en cela que consiste le partenariat public-privé intégrateur.

Il est alors difficile de comprendre pourquoi les entreprises ayant rejoint le Global Compact se refusent (encore) majoritairement à souscrire au projet de Normes des Nations unies sur la responsabilité en matière de droits de l'homme des sociétés transnationales et autres entreprises. L'objectif de ces normes est justement de donner force contraignante aux principes que défend le Global Compact, et donc de contrecarrer légalement les entreprises qui ignorent ce Pacte mondial ou alors qui y ont souscrit «pour faire beau» mais ne le respectent pas. L'argumentation maladroite opposée à ces normes ne peut être comprise que comme une adhérence à l'ancienne dichotomie («plus de liberté, moins d'Etat»). Elle repose sur une alternative fictive entre engagement volontaire et obligation légale, ignorant le fait qu'une entreprise déclarant se reconnaître dans les principes du Global Compact parce qu'elle les juge justes et non pas par simple souci de relations publiques ne saurait logiquement être opposée à leur fixation par écrit. Au contraire, les entreprises qui prennent ce Pacte mondial au sérieux devraient plaider pour que les principes en soient appliqués de manière générale et contraignante, et cela dans leur propre intérêt bien pensé. En effet, ces principes ne nuisent aucunement à leur quête éthiquement intégrée du succès mais les protègent contre la concurrence illicite des moral free-riders dénués de scrupules ${ }^{24}$.

23 Voir G. Kell et J.G. Ruggie, «Global Markets and Social Legitimacy: The Case for the "Global Compact”», Transnational Corporations, vol. 8, n 3, December 1999, p. 105.

24 Voir à ce sujet P. Ulrich, «Der UN Global Compact als Beitrag zu einer "zivilisierten" Weltwirtschaft», Global Compact Deutschland. Jahrbuch 2004, Münster, Macondo, 2004, pp. 84 et suiv. 
Même si l'on ne peut pas compter sur l'adoption à court terme de telles normes contraignantes pour les entreprises transnationales, un tel pas constituerait pour le Global Compact l'étape intermédiaire attendue depuis longtemps: une méthode de sondage permettant d'exclure les entreprises ne cherchant qu'à se parer d'une couche d'un vernis éthique onusien, le blue-washing, abusant de la réputation de l'ONU à de simples fins de relations publiques, sans entreprendre le moindre effort interne d'application des principes bien qu'il s'agisse là d'une condition explicite d'affiliation ${ }^{25}$. On voit donc que le discours sur le «partenariat» des entreprises avec l'ONU est problématique et a soulevé bien des critiques. C'est pour cela qu'il faut établir les critères et références permettant aux entreprises de conserver leur statut de membre. Pourtant, il suffit encore actuellement aux entreprises d'une déclaration d'intention pour obtenir et conserver leur affiliation. Il n'existe toujours pas de contrôle effectif du respect des engagements pris. Pour garantir ce respect - et sur ce point, on peut être entièrement d'accord avec les défenseurs de l'engagement purement volontaire -, il n'est même pas nécessaire d'élaborer une réglementation politique contraignante. Il suffirait dans un premier temps que les entreprises soucieuses de leur réputation bien méritée et donc de la crédibilité de l'initiative obtiennent l'exclusion des «moutons noirs». Le Global Compact de l'ONU est maintenant assez solide pour se permettre d'exclure les membres qui abusent moralement de lui, sans craindre de perdre de son audience. Bien au contraire: l'exclusion d'une entreprise du Global Compact porterait à la réputation de cette dernière un tort durable, une perspective qui devrait encourager l'autocontrôle des membres. Ce qui manque aujourd'hui au Global Compact, c'est exactement ce type de mécanismes qui garantissent la responsabilité de ses membres et en l'absence desquels ce Pacte mondial est condamné à perdre progressivement toute sa crédibilité. Après cinq années d'expérience, le temps nécessaire aux premiers réglages est écoulé. Pour assurer son développement effectif, le Global Compact des Nations unies doit maintenant prendre les mesures permettant de vérifier le respect de ses principes. 


\section{Bibliographie}

Brundtland, G.H., Address by Dr. Gro Harlem Brundtland, Director-General, to the Fifty-Fifth World Health Assembly, Geneva, World Health Organization, 13 May 2002, <www.who.int/genomics/ speeches/en> (dernière consultation: 16 janvier 2005).

DwORKIN, R., Bürgerrechte ernst genommen, Frankfurt a. M., Suhrkamp, 1984.

FITSCHEN, T., Der «Global Compact» als Zielvorgabe für verantwortungsvolles Unternehmertum. Idee mit Zukunft oder Irrweg für die Vereinten Nationen?, Diskussionspapier, $\mathrm{n}^{\circ}$ 04-8, Wittenberg, Wittenberg-Zentrum für Globale Ethik, 2004.

KANT, I., «Beantwortung der Frage: Was ist Aufklärung?», in Weischedel, W. (dir.), Immanuel Kant Werkausgabe, vol. 11, 6 édition, Frankfurt a. M., Suhrkamp, 1982, pp. 53-61.

Kell, G., Ruggie, J.G., "Global Markets and Social Legitimacy: The Case for the "Global Compact" », Transnational Corporations, vol. 8, n 3, December 1999, pp. 101-120.

Lunau, Y., Wettstein, F., Die soziale Verantwortung der Wirtschaft. Was Bürger von Unternehmungen erwarten, Bern; Stuttgart; Wien, Haupt, 2004.

MaAк, T., Die Wirtschaft der Bürgergesellschaft, Bern; Stuttgart; Wien, Haupt, 1999.

Mirow T., «Public-Private Partnership - eine notwendige Strategie zur Entlastung des Staates», in Budäus, D., EICHhoRn, P. (dir.), Public-Private Partnership. Neue Formen öffentlicher Aufgabenerfüllung, Baden-Baden, Nomos Verlagsgesellschaft, 1997, pp. 13-23.

NigGLI, P., Nach der Globalisierung. Entwicklungspolitik im 21. Jahrhundert, Zürich, Rotpunktverlag, 2004.

Richter, J., Public-Private Partnerships and International Health Policy Making: How Can Public Interests be Safeguarded?, Helsinki, Ministry for Foreign Affairs of Finland, 2004.

—, «We the Peoples» or «We the Corporations»? Critical Reflections on UN-business «Partnerships», Geneva, International Baby Food Action Network (IBFAN); Geneva Infant Feeding Association (GIFA), 2003.

Savas, E.S., Privatization and Public-Private Partnerships, New York; London, Chatham House Publishers, 2000.

SEN, A., Un nouveau modèle économique. Développement, justice, liberté, Paris, Odile Jacob, 1999 (trad. fr. de Development as Freedom).

ThIELEMAnN, U., Freiheit unter den Bedingungen des Marktes. Oder doch gegenüber der Marktlogik? Vom verfehlten Umgang mit Sachzwängen, Berichte des Instituts für Wirtschaftsethik, $\mathrm{n}^{\circ} 101$, St. Gallen, Institut für Wirtschaftsethik, 2004.

Ulrich, P., Der entzauberte Markt. Eine wirtschaftsethische Orientierung, Freiburg i. B., Herder, 2002.

—, «Der UN Global Compact als Beitrag zu einer "zivilisierten” Weltwirtschaft», Global Compact Deutschland. Jahrbuch 2004, Münster, Macondo, 2004, pp. 84-86.

—, Die Grossunternehmung als quasi-öffentliche Institution. Eine politische Theorie der Unternehmung, Stuttgart, C. E. Poeschel, 1977.

—, Integrative Wirtschaftsethik, Grundlagen einer lebensdienlichen Ökonomie, $3^{\mathrm{e}}$ édition, Bern; Stuttgart; Wien, Haupt, 2001.

—, «Was ist "gute" sozio-ökonomische Entwicklung? Eine wirtschaftsethische Perspektive», Zeitschrift für Wirtschafts- und Unternehmensethik, vol. 5, nº 1, 2004, pp. 8-22.

WetTsteIn, F., Die «unsichtbare Hand» des Wassermarktes. Zur Privatisierungs- und Wettbewerbslogik im Wasserversorgungssektor, Berichte des Instituts für Wirtschaftsethik, n 103 , St. Gallen, Institut für Wirtschaftsethik, 2004. 


\section{Annexe}

\section{Les dix principes du Pacte mondial (Global Compact) de I'ONU}

Le Pacte mondial engage les dirigeants des entreprises à "embrasser, promouvoir et faire respecter" un ensemble de valeurs fondamentales dans le domaine des droits de l'homme, des normes du travail, de l'environnement et de la lutte contre la corruption.

Ces dix principes sont inspirés de:

๑ la Déclaration universelle des droits de l'homme;

- la Déclaration relative aux principes et droits fondamentaux au travail (Organisation internationale du travail):

- la Déclaration de Rio sur l'environnement et le développement;

- la Convention des Nations unies contre la corruption.

\section{Droits de l'homme}

Principe 1: Les entreprises doivent promouvoir et respecter les droits de l'homme reconnus sur le plan international.

Principe 2: Les entreprises ne doivent pas se faire complices de violations des droits fondamentaux.

\section{Normes de travail}

Principe 3: Les entreprises devraient respecter l'exercice de la liberté d'association et reconnaître le droit à la négociation collective.

Principe 4: Elimination de toutes les formes de travail forcé et obligatoire.

Principe 5: Abolition effective du travail des enfants.

Principe 6: Elimination de la discrimination en matière d'emploi et d'exercice d'une profession.

\section{Environnement}

Principe 7: Promouvoir une approche prudente des grands problèmes touchant l'environnement.

Principe 8: Prendre des initiatives en faveur de pratiques environnementales plus responsables.

Principe 9: Encourager la mise au point et la diffusion de technologies respectueuses de l'environnement.

\section{Lutte contre la corruption}

Principe 10: Les entreprises sont invitées à agir contre la corruption sous toutes ses formes, y compris l'extorsion de fonds et les pots-de-vin. 